\title{
AUTOMATIC EXTRACTION OF MANGROVE VEGETATION FROM OPTICAL SATELLITE DATA
}

\author{
Sushma Reddy ${ }^{\mathrm{a}}$, Mayank Agrawal ${ }^{\mathrm{a}}$, Ram Chandra Prasad ${ }^{\mathrm{b}}$ \\ ${ }^{a}$ Lab for Spatial Informatics, IIIT Hyderabad (sushma.reddy, mayank.agrawal)@ research.iiit.ac.in \\ ${ }^{\mathrm{b}}$ Lab for Spatial Informatics, IIIT Hyderabad, rcprasad@iiit.ac.in
}

Commission VI, WG VI/4

KEY WORDS: Mangroves, LISS , Landsat 8, segmentation, pixel value, gabor filtering, Otsus method

\begin{abstract}
:
Mangrove, the intertidal halophytic vegetation, are one of the most significant and diverse ecosystem in the world. They protect the coast from sea erosion and other natural disasters like tsunami and cyclone. In view of their increased destruction and degradation in the current scenario, mapping of this vegetation is at priority. Globally researchers mapped mangrove vegetation using visual interpretation method or digital classification approaches or a combination of both (hybrid) approaches using varied spatial and spectral data sets. In the recent past techniques have been developed to extract these coastal vegetation automatically using varied algorithms. In the current study we tried to delineate mangrove vegetation using LISS III and Landsat 8 data sets for selected locations of Andaman and Nicobar islands. Towards this we made an attempt to use segmentation method, that characterize the mangrove vegetation based on their tone and the texture and the pixel based classification method, where the mangroves are identified based on their pixel values. The results obtained from the both approaches are validated using maps available for the region selected and obtained better accuracy with respect to their delineation. The main focus of this paper is simplicity of the methods and the availability of the data on which these methods are applied as these data (Landsat) are readily available for many regions. Our methods are very flexible and can be applied on any region.
\end{abstract}

\section{INTRODUCTION}

Mangroves are halophytes (i.e., a plant adapted to growing in saline conditions) which grow in loose wet soils of brackish to saline estuaries and shorelines in the tropics and subtropics (Heumann, 2011, Aheto et al., 2011, Ashton et al., 1999, Tomlinson and Tomlinson, 1994). They cover up to $75 \%$ of the worlds tropical coastlines (Spalding et al., 1997). A large amount of fauna like shrimp, fishes, snakes, crabs, mammals, insects and microorganisms are found in mangroves ecosystem.Mangrove forests plays an important role. It is being used by people as their food resource, firewood and other products(Prasad et al., 2010). The ecosystem products and services that mangroves provide include protecting the coastline from tidal waves and storm surges; behave as biological filters in polluted coastal areas; supporting aquatic foodchains; and shielding a large number of juvenile aquatic organisms (Vaiphasa et al, 2006). In the current scenario different types of human intervention and natural disturbances, carried out in mangrove forest area like shrimp farming, industrial activities and settlements, causing reduction and damage to mangrove forests. These repercussions have subsequently drawn considerable attention to the conservation and management of this unique estuarine ecosystem (Secretariat, 2004). So to preserve them and to maintain them, an accurate spatial information of mangroves is required. The traditional way is field survey, but it is very difficult. to access all areas of Mangroves.Also it takes a huge amount of time.

In India, the area under Mangrove is $4740 \mathrm{Sq} . \mathrm{km}$ as per the estimate of the Forest survey of India (2015). Out of this, 617 sq. $\mathrm{km}$ of vegetation of Mangrove occur in Andaman and Nicobar Islands. The mangrove vegetation of the islands constitute $7.5 \%$ of the total forest area. Mangroves in these areas is mostly found in creeks, backwater and muddy shores.

So in this paper we are trying to find out the ways by which we

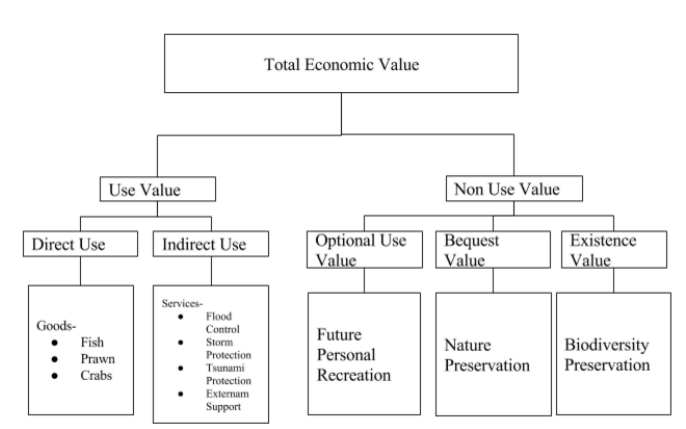

Figure 1: Figure describes different economic uses of the Mangroves.

can easily figure out the mangroves in satellite images. Focus of this paper is finding the optimum computationally intensive method for their detection of mangroves.

Various types of remotely sensed imagery and processing methods have been to identify and map the mangrove. An object-based method for mapping and change analysis in mangrove ecosystems was used (Conchedda et al., 2008).Also for example, (Green et al., 1998) used principal component analysis and band ratios for processing multi-spectral images, and (Saito et al., 2003) compared different classifiers for SPOT XS and ASTER Terra images. More recently, a Bayesian probability method incorporating ecological data was used to develop a post-classifier for mangroves (Vaiphasa, 2006). Many studies on mangroves were done using hyperspectral data too(Kamal and Phinn, 2011, Zhang et al., 2014).

In this paper the focus is on the simplicity of the methods and readily availability of the data so that anyone can use it. The data used here is LISS III and Landsat 8 data which is freely avail- 


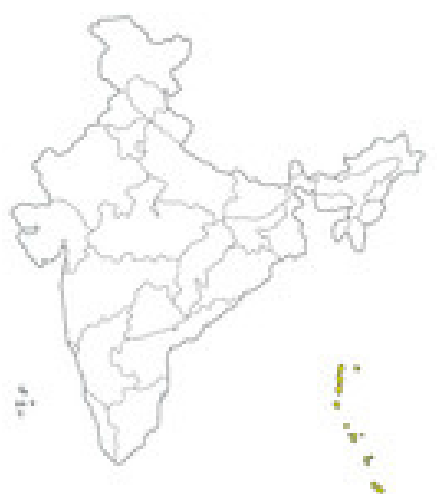

Figure 2: Map showing Andaman and Nicobar Islands in green color.

\begin{tabular}{|c|c|c|}
\hline Band & Spectral Band $(\mu \mathrm{m})$ & Resolution $(\mathrm{m})$ \\
\hline 2 (Green) & $0.52-0.59$ & 23 \\
\hline 3 (Red) & $0.62-0.68$ & 23 \\
\hline 4 (NIR) & $0.77-0.86$ & 23 \\
\hline 5 (SWIR) & $1.55-1.70$ & 23 \\
\hline
\end{tabular}

Figure 3: Characteristics of LISS-III sensor.

able for most of the areas and anyone can access it. The methods used here are K-means clustering, SVM classifiers, Gabor filtering, Otsu's threshold method and Texture and color segmentation. The results of our work include the images where the Mangrove area is highlighted in the provided Satellite Image. Since there is nothing to mathematically check the accuracy so we are checking it with visual interpretations of the experts who had done field survey for this.

\section{STUDY AREA AND SATELLITE DATA}

\subsection{Study Site}

The study area is the Mangrove forest in Andaman and Nicobar Islands which is extended from Latitude $-6^{\circ}$ to $14^{\circ}$ North, Longitude $-92^{\circ}$ to $94^{\circ}$ East. Andaman and Nicobar islands are one of the seven territories of India. The total area covered by these islands is about 7950sq.km. Of this total area more than $80 \%$ of the land is covered with various vegetation. And more than $20 \%$ of the land division is mangroves. About one fifths of the total mangrove vegetation present in India is found in Andaman and Nicobar islands.

\subsection{Satellite data}

The optical data of LISS-III sensor of 2011 and Landsat 8 sensor of 2011 are used for this study. LISS-III satellite data consists of four spectral bands ranging from 0.52 to $1.70 \mathrm{~m}$ and all are of $23.5 \mathrm{~m}$ spatial resolution. And Landsat 8 consists of nine spectral bands with spatial resolution of $30 \mathrm{~m}$. Landsat TM data and ASTER DEM data acquired from USGS are also used in the study.

False Color Composition $(4,3,2)$ of LISS-III data is used for recognizing mangroves. Various kinds of vegetation, including mangroves, produce a high peak in the NIR band and low in the red

\begin{tabular}{|c|c|c|}
\hline Bands & Wavelength $(\mu \mathrm{m})$ & Resolution $(\mathrm{m})$ \\
\hline Band 1 - Coastal aerosol & $0.43-0.45$ & 30 \\
\hline Band 2 - Blue & $0.45-0.51$ & 30 \\
\hline Band 3 - Green & $0.53-0.59$ & 30 \\
\hline Band 4 - Red & $0.64-0.67$ & 30 \\
\hline Band 5 - Near Infrared (NIR) & $0.85-0.88$ & 30 \\
\hline Band 9 - Cirrus & $1.36-1.38$ & 30 \\
\hline Natural Color Image & & 30 \\
\hline
\end{tabular}

Figure 4: Characteristics of Landsat 8 sensor.

and green region of spectrum, with reference to the absorption of the chlorophyll. Chlorophyll contents in mangroves reflect high in the Near Infrared (NIR) band of the electromagnetic spectrum. Hence mangroves could be distinguished from other land cover types in images with bright red colour pixels and smooth texture if they are densely distributed. The sparsely distributed mangrove appears in pinkish red colour. And True Color composite $(1,2,3)$ of Landsat 8 data is used in this particular study.

\section{METHODS}

Different vegetation have different spectral characteristics based on the chlorophyll content in the leaves, water content, weather conditions, etc. For vegetation type mapping the commonly used elements are tone (color) and texture. In this paper we will try to recognize and extract the mangroves vegetation based on the false color composite of satellite images of LISS- III using color and texture elements and True color composite of Landsat 8 using the pixels values. Mangroves have dark red velvety tone and smooth texture.

Various methods to recognize mangroves have been implemented and their time complexity and accuracy are studied. The methods implemented in this study using LISS- III data are K-means clustering, Gabor filtering, Otsu's threshold method and Texture and color segmentation. SVM classification and Color based pixel classification are implemented using the Landsat 8 images. All the classifications done are Supervised except K means. All these methods along with the observations are discussed below.

\subsection{K means clustering}

It is a process of partitioning a collection of data into a small number of clusters. Partitioning data into clusters is done by measuring certain features of data. In general, we have $\mathrm{n}$ data points $\mathrm{Xi}$, $\mathrm{i}=1$...n and we need to be partition them into k clusters. K-means is one of the simplest unsupervised learning algorithms that solve the clustering problem. Initially, $\mathrm{K}$ random centroids are chosen, where $\mathrm{K}$ is the number of desired clusters defined by the user. Each point is then assigned to a nearest centroid and the collection of points assigned to a centroid form a cluster. Then centroid re-calculated for the points in the cluster and this process goes on stops when the points no more change their clusters.

Algorithmic Steps for K-means Clustering:

1. Initialize the centres of the required number of clusters.

2. Attribute the closest cluster to each data point. (The feature considered is color) 


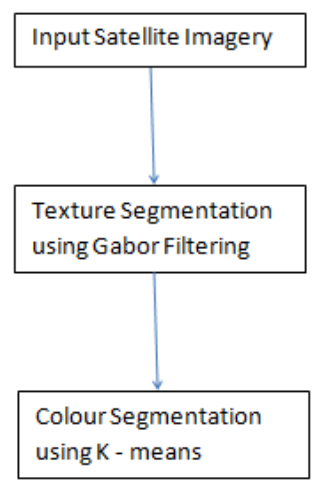

Figure 5: Work flow for Gabor filtering

3. Recompiling the centroids.

4. Goto step1 if points moved from one cluster to other. Else stop.

5. Label the clusters and segment.

\subsection{Gabor Filtering}

Gabor filters can perform in both the spatial and frequency domain. Considering the spatial domain and frequency domain values is very important in texture segmentation. So, Gabor filtering is widely used in texture segmentation in images. This techniques gives finer distinction between the textures in an image. Gabor filter is basically a Gaussian function modulated by complex sinusoidal of frequency and orientation(Bharathi P.T, 2013).

In spatial domain, Gabor function is a sinusoidal modulated function. The $2 \mathrm{D}$ gaussian curve $\mathrm{g}(\mathrm{x}, \mathrm{y})$ is given as follows

$g(x, y)=\frac{1}{2 \pi \sigma_{x} \sigma_{y}} \exp \left\{-\frac{1}{2}\left[\left(\frac{x^{2}}{\sigma_{x}^{2}}\right)\left(\frac{y^{2}}{\sigma_{y}^{2}}\right)\right]\right\} \cdot \cos \left(2 \pi u_{0} x\right)$

where $\quad u_{0}=$ modulating frequency

$\sigma_{x}, \sigma_{y}=$ spatial extent and bandwidth of filter

And in the spatial-frequency domain, the Gabor filter becomes two shifted Gaussians at the location of the modulating frequency(Khaled Hammouda, n.d.). The equation of the 2D frequency curve is given by

In spatial domain, Gabor function is a sinusoidal modulated function. The $2 \mathrm{D}$ gaussian curve $\mathrm{g}(\mathrm{x}, \mathrm{y})$ is given as follows

$$
\begin{aligned}
H(u, v) & =\exp \left\{-2 \pi^{2}\left[\sigma_{x}^{2}\left(u-u_{0}\right)+\sigma_{y}^{2} v^{2}\right]\right\} \\
& +\exp \left\{-2 \pi^{2}\left[\sigma_{x}^{2}\left(u+u_{0}\right)+\sigma_{y}^{2} v^{2}\right]\right\}
\end{aligned}
$$

The work flow for this technique is mentioned in the Figure 2. The algorithmic steps taken for texture segmentation are clearly explained in the paper(Bharathi P.T, 2013). The output obtained after gabor filtering is image with different colors indicating different textures in the image. This image is given as input to the $\mathrm{K}$ means clusteing(3.1), for color based classification to extract the image which only contains mangroves.

\subsection{Otsu's Method}

The Landsat TM data and ASTER DEM data acquired from USGS are used in this study. Mangrove forests are mostly distributed in the coastal wetland areas between land and sea. By analysis it is found that mangroves are found in the areas which have elevation less than $30 \mathrm{~m}$ [otsu paper]. So, based on these results the areas in the ASTER DEM which have elevation higher than $30 \mathrm{~m}$ are eliminated as non mangroves.

The reflectance of vegetation in the short wavelength infrared (SWIR) spectrum was attributed to the water absorption and scattering (Woolley, 1971). Mangroves has very less intercellular air spaces compared to other vegetation. So, they can be attributed to weak scattering.

Otsus threshold $\mathrm{T}$ is used for classifying mangroves and nonmangrove regions. This is a non-parametric and unsupervised classification technique. $\mathrm{T}$ is defined as

$$
T=\frac{\left(\mu^{\prime} w(T)-\mu(T)\right)^{2}}{w(T) \mu(T)} e 4
$$

where

$$
\begin{aligned}
& \mu^{\prime}=\sum i * p_{i}, p_{i} \text { is the probability } \\
& \text { of pixels with grey value } \mathrm{i} \\
& w(T)=\sum_{i=0}^{T} p_{i}, \mu(T)=\sum_{i=T+1}^{255} p_{i}
\end{aligned}
$$

The pixels with Digital Number value less than $\mathrm{T}$ are considered as mangroves.

\subsection{Texture and Color segmentation}

From the previously explained methods it is clear that considering any one of the elements tone or texture alone doesnt give better results. So to effectively extract the mangroves in the optical images by considering both the elements tone and texture we have implemented the following method. Texture quantifies gray level differences in an image. In the first step the image is segmented by extracting the texture features of the image. Various methods perform well in extracting the texture features on the gray values of image GLCM, GCM, Autocorrelation, two dimensional spatial filtering, etc. In our study we have used the 2D spatial filtering method. This operation is similar to filtering. It transforms an image into a feature image.

Computing texture images involves four steps

1. Select a window size and also texture measure to find the texture features (say $3 \times 3$ ).

2. Centre the window at each pixel $(i, j)$ in the image.

3. Compute the texture measure.

4. Assign the computed value to the centre pixel $(i, j)$ in a new image of the same size.

The texture measure considered is the sum of the differences of mean of the intensity values in $3 \times 3$ mask to intensity values of its $3 \times 3$ neighbours.

$$
\text { SumofDifferences }=\sum_{i}^{w_{i}} \sum_{j}^{w_{j}}|f(i, j)-\mu|
$$


where

$$
\begin{aligned}
& \mu=\text { mean gray level value of the entire window } \\
& f(i, j)=\text { gray level value of the root pixel of a } \\
& 3 \times 3 \text { pixel window }
\end{aligned}
$$

The resultant image obtained is a texture featured image. A $3 \times 3$ template of mangroves is considered and the same texture measure is found as done above. Now, by taking the L2 norm all the features of the input image and the template and thresholding it, we obtained an image with all the forests type containing same and nearly same texture as that of mangroves.

For colour segmentation of the image, the output of texture segmentation is taken and the colour feature image is calculated similarly as in the case of texture segmentation by considering the colour values instead of intensity values. By taking the L2 norm and thresholding the final result is obtained.

\subsection{Color based Pixel Classification}

Each band gives the reflectance of objects in different wavelength and they have gray scale values. Based on the visual interpretation of the experts, the bands in Landsat 8 data which are useful to obtain the required result are chosen. Then by thresholding the reflectance values of the selected bands. The thresholding is done by trial and error method. Then by finding the intersection of individual results of the selected bands we obtain the final result which shows the areas where the mangroves are located. It is observed that the input data is prone to noise due to clouds. So, preprocessing is done using band 9 (cirrus) to remove the clouds from the input data.

\subsection{SVM Classification}

support vector machines (SVM) is used in machine learning. it is a model for the supervised learning with associated learning algorithms of analyzing the data and recognizing the patterns, used for classification and regression analysis. If a set of training examples are given, each marked for categorized to one of two categories, an SVM training algorithm builds a model that categorizes new examples into one category or the other, making it a non-probabilistic binary linear classifier.(wikipedia,2016)

Computing the (soft-margin) SVM classifier amounts to minimizing an expression of the form

$$
\left[\frac{1}{n} \sum_{i=1}^{n} \max \left(0,1-y_{i}\left(w \cdot x_{i}+b\right)\right)\right]+\lambda\|w\|^{2}
$$

\section{where $\quad \lambda$ determines the tradeoff between}

$$
\begin{aligned}
& \text { increasing the margin-size and ensuring that } \\
& \text { the } x_{i} \text { lie on the correct side of the margin. } \\
& y_{i}=\text { either } 1 \text { or } 1 \text {, each indicating the class } \\
& \text { to which the point } x_{i} \text { belongs. } \\
& w=\text { normal vector to the hyperplane. }
\end{aligned}
$$

The true color composite (1,2,3 bands) or Natural image is chosen for SVM classification. The training samples are 3 dimensional with its components being red, green and blue reflectance values for the classifier to learn the mangroves. After training the SVM classifier, the test input satellite image is provided to the classifier. The pixels of the true color composite of this input are classified into the two classes (Mangroves and non mangroves). The pixels in the Mangroves class are shown in the result.

\subsection{Pixel thresholding on Natural color Image}

By the observations from the result obtained from the SVM classification it is found that a specific range of RGB pixels values are mangroves. This range is applicable for all the Landsat 8 images. The results obtained are accurate compared to the other methods discussed for Landsat 8 images. To extract mangroves, we have to only look for these values and mark those values. The simplest thresholding methods replace each pixel in an image with a black pixel if the image intensity $\left.I_{(} i, j\right)$ is less than some fixed constant $\mathrm{T}$ (that is, $I(i, j)<T$ ), or a white pixel if the image intensity is greater than that constant.(wikipedia,2016) For pixel thresholding, Spatial methods are used which take into consideration the correlation between pixels.

\section{RESULTS AND DISCUSSION}

The results given in the paper are using the Northern Andaman for LISS-III data and the whole Andaman and Nicobar region for Landsat 8 data. But the methods mentioned work for any region. For the K-Means Clustering, 6(b) though the algorithm is simple, easy to understand and fast, there are many disadvantages of this algorithm.

1. As the classification is only based on color, all the forest types which have same tone similar to mangroves are also classified into the same class.

2. Number of classes should be defined a priori and also the change in number of classes defined affects the classification.

3. Randomly chosen cluster centres may not lead us to a fruitful result.

Considering these drawbacks next method, Gabor Filtering is implemented. The output 6(c) obtained after Gabor filtering is classified by color based segmentation using K-means clustering. But the results obtained here are not satisfactory. Some of the drawbacks observed in this method are

1. Many other areas which are not mangroves are included in the same class of Mangroves.

2. The results are not always constant when the algorithm is run for different times. When the algorithm is run for the second time the putput is $6(\mathrm{~d})$

3. It is not possible to identify to which class Mangroves belong automatically.

Taking into consideration all the above drawbacks, the next method of texture and color segmentation is is implemented. The results obtained after texture segmentation is $6(\mathrm{~g})$. For colour segmentation of the image, the output of texture segmentation is taken and the colour feature image is found similarly as in the case of texture segmentation by considering the colour values instead of intensity values. By taking the L2 norm and thresholding the final result obtained is 6(h). The final result only consists of mangroves, so better when compared to other two methods. For the Otsus method, the result is 6(e). The result seems to be good but this always depends on the threshold chosen.

Now, with the Landsat 8 data, the results obtained using classification based on the band images are observed to be not accurate 
compared to the required results.6(j) Also there are many other drawbacks using this method. It is computationally intensive as we are working on many bands and each band is of high spatial resolution. Also choosing a bad threshold leads to over or under classification. So we moved to the next method based on SVM classification.

For SVM classification, the training samples chosen are similar to $6(\mathrm{k}), 6(\mathrm{l})$.In this method, it is observed that some of the pixels are alone in the result. To remove this salt and pepper noise, post processing of the results is done which eliminates the pixel regions which have area less than a threshold. 6(m) From this SVM classification, we can get to know about the threshold which will give us the result. In the next method of the pixel threshold on the Natural color image, we will use the thresholds obtained after the SVM classification and we can use these thresholds for all the Natural color images of this satellites for the detection of the Mangroves. We dont have to run the SVM learning every time. Once it is used for finding the thresholds, all the data of same resolution and taken by same satellite can be classified for the detection of the mangrove6(n).The ranges we got for this particular case of Andaman and Nicobar is (red $<70,120<$ green $<140$, blue $<100)$.

\section{CONCLUSION AND FUTURE WORK}

For K means number of classes should be known before hand and cant identify mangroves exactly. Gabor filter doesnt give the same segmented classes every time. Texture and color segmentation method gives better results but depends on the template chosen. But comparatively the results obtained in third method are satisfactory and more generalized. The time taken to for the algorithm to run is around 1-2 seconds. So, this can be declared to be more efficient and faster than other two methods. The results of Otsus method are not mathematically assessed. But the results seem to be good when visually verified with the vector data of the mangroves in Andaman and Nicobar Islands. In future more generalized texture extraction techniques can be used to obtain better results in case of LISS III data.

Pixel based classification is feasible but it involves a lot of try and test. The knowledge about the classification can be only by trying with different values and thus a lot of time is consumed. So in future we can classify each feature in each band thus the classification will become easy. The results obtained from SVM seem to be quite acceptable. In the future, instead of SVM, we can use SIFT features for the extraction of the features from the image and then classify using these features. This will be more accurate and more closer to the required.

All the methods mentioned in the paper are experimented with both Landsat 8 and LISS-III optical data and the results are satisfactory. 


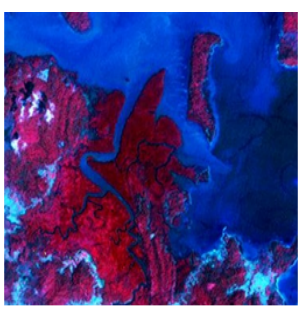

(a) Input LISS 3 image FCC

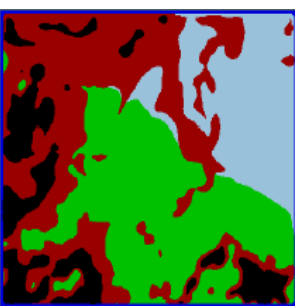

(c) One of the outputs from Gabor Filtering

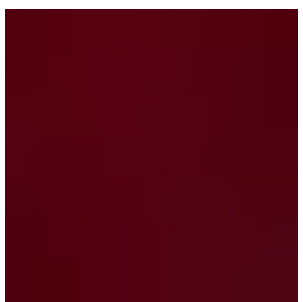

(f) $3 \times 3$ pixel template for Mangroves

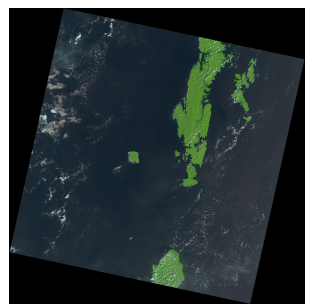

(i) Input Landsat 8 TCC

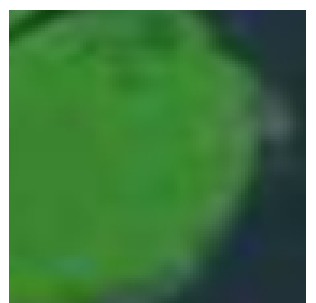

(k) Training sample for SVM

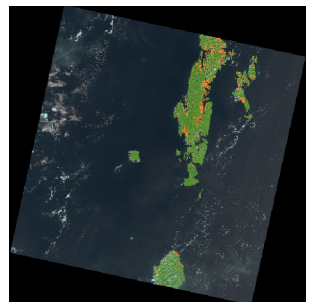

(m) Output for SVM

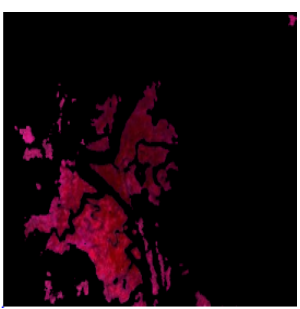

(b) Output of K means

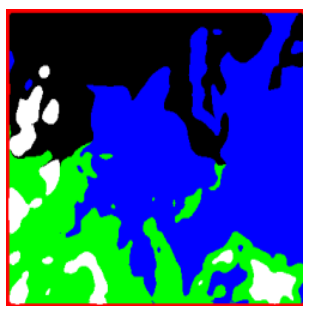

(d) Another output from Gabor Filtering

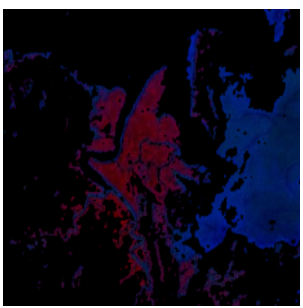

(g) Ouput after Texture segmentation (e) Output method

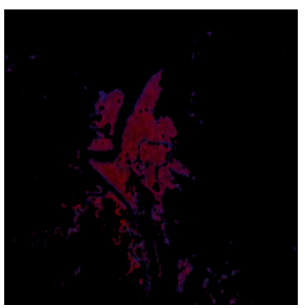

(h) Final output after Color segmentation

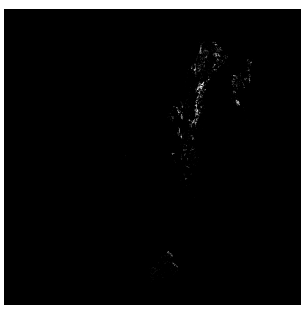

(j) Output for Color based pixel classification

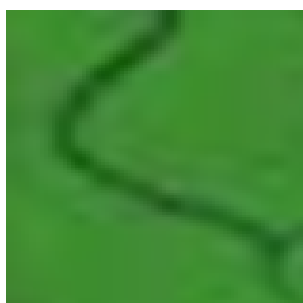

(1) Training sample for SVM

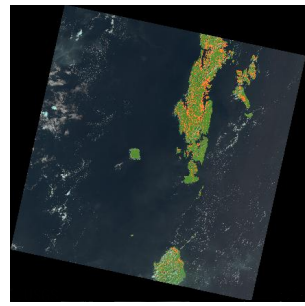

(n) Highlighting Mangroves by the defined range

Figure 6: Input images and the results obtained in various methods discussed. 


\section{ACKNOWLEDGEMENTS}

We thank USGS for making available data for free.

\section{References}

Aheto, D., Mensah, E., Aggrey-Fynn, J., Obodai, E., Mensah, C., Okyere, I. and Aheto, S., 2011. Spatio-temporal analysis of two coastal wetland systems in ghana: Addressing ecosystem vulnerability and implications for fisheries development in the context of climate and land use changes. Archives of Applied Science Research 3(3), pp. 499-513.

Ashton, E., Hogarth, P. and Ormond, R., 1999. Breakdown of mangrove leaf litter in a managed mangrove forest in peninsular malaysia. Hydrobiologia 413, pp. 77-88.

Bharathi P.T, D. P. S., 2013. Texture based color segmentation for infrared river ice images using $\mathrm{k}$ - means clustering. International Conference on Signal Processing, Image Processing and Pattern Recognition.

Conchedda, G., Durieux, L. and Mayaux, P., 2008. An objectbased method for mapping and change analysis in mangrove ecosystems. ISPRS Journal of Photogrammetry and Remote Sensing 63(5), pp. 578-589.

Green, E. P., Clark, C. D., Mumby, P. J., Edwards, A. J. and Ellis, A., 1998. Remote sensing techniques for mangrove mapping. International Journal of Remote Sensing 19(5), pp. 935956.

Heumann, B. W., 2011. Satellite remote sensing of mangrove forests: Recent advances and future opportunities. Progress in Physical Geography 35(1), pp. 87-108.

Kamal, M. and Phinn, S., 2011. Hyperspectral data for mangrove species mapping: A comparison of pixel-based and object-based approach. Remote Sensing 3(10), pp. 2222-2242.

Khaled Hammouda, P. E. J., n.d. Texture segmentation using gabor filters. University of Waterloo, Ontario, Canada.

Prasad, P. R. C., Nagabhatla, N., Reddy, C., Gupta, S., Rajan, K., Raza, S. and Dutt, C., 2010. Assessing forest canopy closure in a geospatial medium to address management concerns for tropical islandssoutheast asia. Environmental monitoring and assessment 160(1-4), pp. 541-553.

Saito, H., Bellan, M., Al-Habshi, A., Aizpuru, M. and Blasco, F., 2003. Mangrove research and coastal ecosystem studies with spot- 4 hrvir and terra aster in the arabian gulf. International Journal of Remote Sensing 24(21), pp. 4073-4092.

Secretariat, R. C., 2004. The ramsar convention manual: a guide to the convention on wetlands (ramsar, iran, 1971). Ramsar Convention Secretariat Gland, Switzerland.

Spalding, M., Blasco, F. and Field, C., 1997. World mangrove atlas.

Tomlinson, P. B. and Tomlinson, P. B., 1994. The botany of mangroves. Cambridge University Press.

Vaiphasa, C., 2006. Remote sensing techniques for mangrove mapping. Citeseer.

Woolley, J., 1971. Reflectance and transmittance of light by leaves. Plant Physiol.

Zhang, C., Kovacs, J. M., Liu, Y., Flores-Verdugo, F. and Floresde Santiago, F., 2014. Separating mangrove species and conditions using laboratory hyperspectral data: A case study of a degraded mangrove forest of the mexican pacific. Remote Sensing 6(12), pp. 11673-11688.
Sahni, K.C., 1958. Mangrove forests in the Andaman and Nicobar Islands.Indian Forester, 84(9), pp.554-562.

Sremongkontip, S., Hussin, Y.A., Groenindijk, L. and Detection, C., 2000. Detecting changes in the mangrove forests of southern Thailand using remotely sensed data and gis. International Archives of Photogrammetry and Remote Sensing, 33(1), pp.567574.

Florida Museum of Natural History., 2016. Importance of Mangroves. https://www.flmnh.ufl.edu/southflorida/

habitats/mangroves/importance-mangroves.

Vidhya, R., Vijayasekaran, D., Farook, M.A., Jai, S., Rohini, M. and Sinduja, A., 2014. Improved Classification of Mangroves Health Status Using Hyperspectral Remote Sensing Data. The International Archives of Photogrammetry, Remote Sensing and Spatial Information Sciences, 40(8), p.667.

National Portal of India., 2016. Environment and forests. http://forest.and.nic.in/ 